\title{
DIGITIZED SPARK CHAMBER FOR SPACE SCIENCE EXPERIMENTS
}

\author{
BY \\ R. W. ROSS \\ C. H. EHRMANN \\ C. E. FICHTEL \\ D. A. KNIFFEN
}

GPO PRICE

s

CFSTI PRICE(S) \$

Hard copy (HC)

Microfiche (MF)

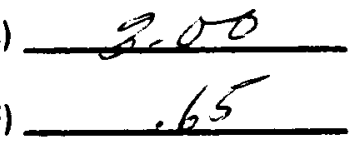

NOVEMBER 1966

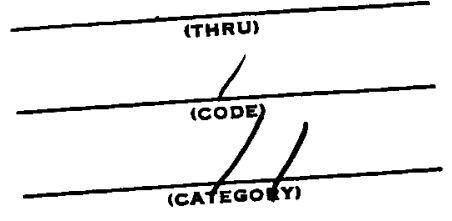




\section{DIGITIZED SPARK CHAMBER \\ FOR}

SPACE SCIENCE EXPERIMENTS

R. W. Ross, C. H. Ehrmann, C. E. Fichtel, and D. A. Kniffen 


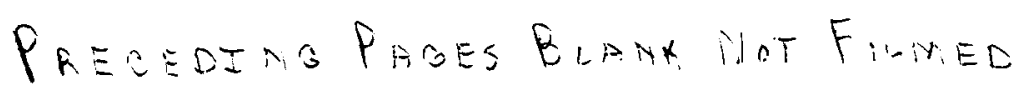

DIGITIZED SPARK CHAMBER
FOR

SPACE SCIENCE EXPERIMENTS

R. W. Ross, C. H. Ehrmann, C. E. Fichtel, and D. A. Kniffen

\begin{abstract}
A digitized spark chamber has been developed for gamma-ray and charged particle experiments on balloons and satellites. The spark chamber is assembled from modular units, which are stacked to the desired number of levels. Each module consists of parallel spark planes formed from two orthogonal wire grids. Memory cores, strung on the wires of both grids, record the X-Y coordinate of the spark. Factors affecting the operating characteristics of these modules are discussed.

A gamma-ray detector of 32 module levels has been assembled and balloonflown in a search for cosmic gamma-ray point sources. The construction of this detector is described and its performance briefly discussed.
\end{abstract}




\section{INTRODUCTION}

The value of spark chambers in nuclear physics experiments is a long established fact, and these versatile charged particle detectors are rapidly finding their way into various cosmic-ray studies where their pictorial nature may not only give excellent directional information, but may also aid in the sifting out of false or spurious events. The development of digitized, wiregrid spark chamber modules with narrow separation, good sparking efficiency, and good spatial resolution has made possible the assembly of a variety of space science experiments for balloon or satellite flight-recovery being made unnecessary by the electronic digitization. Several of these modult s may be stacked in a compact array with a large angle of acceptance and minimum scattering material, while retaining the redundancy advantages of many planes. In addition to experiments on charged particles, those on neutrals may be accommodated by inclusion of interaction plates in a recess provided between modules.

\section{MODULE CONSTRUCTION}

Shown in Figure 1 is a typical X-Y spark chamber module formed by stretching two orthogonal sets of 128 wires on either side of a six by six inch frame of glass-bonded mica. The six mil beryllium-copper wires, .047 inches apart, are ultrasonically welded to copper posts embedded in the frame on one end. They are each tensioned to one pound and welded to the post at the opposite end. The welds are then solder covered to improve the peeling resistance of the joint. Individual wires attached in this manner deviate only two mils from the nominal separation of planes of about .080 inch.

Each spark wire is extended through its memory core to terminate on a common buss along the edge of the core shelf. For certain applications, which will be discussed later, a resistor is placed in the spark wire between the welded pin and the core. The cores on each shelf are also threaded with a sense wire which, in conjunction with a third wire - the drive wire, is necessary to read the memory. Since a sizable potential difference may exist between the spark wires and the readout wires, doubly insulated Nylclad wire is used on all wires passing through the core aperture and a thin RTV compound is applied to the cores as a further precaution. 


\section{MODULE OPERATING CHARACTERISTICS}

\section{Efficiency}

It has been found that these modules of small plane separation (.080 inch) require considerable care in gas purity, choice of mixture and in the characteristics of supporting high voltage circuitry. The use of pure neon, in the case where the greatest critical distance ${ }^{1}$ is only a millimeter or two, requires very high values of applied electric field. By the addition of a small quantity of argon, utilizing the Penning effect on metastable states of neon, a considerable reduction in working voltage and a less stringent requirement on high voltage pulse rise-time are obtained.

A further consequence of a narrow gap is the reduction of the maximum obtainable sparking efficiency, which is essentially the probability of at least one Poisson-distributed free electron available within the gap after passage of the ionizing particle. As may be seen in Figure 2a, this may be offset by an increase in pressure. At atmospheric pressure the "plateau" efficiency is around $85 \%$ but with a spark gas pressure of 25 p.s.i. an improvement of nearly $10 \%$ is obtainable.

\section{Spark Spreading}

The tendency of the spark discharge to spread over several adjacent wires may also be improved by an increase in operating pressure. Referring to Figure $2 b$, the spark spreading is largely determined by the voltage applied above threshold. The sharper knee in the efficiency curves at higher pressure allows a smaller overvoltage, hence a lower spreading. These spreading curves were taken using a standard module incorporating $100 \mathrm{ohm}$ resistors in series with the cathode grid wires for improvement in multiple track efficiency. In applications not requiring high multiple efficiency, average spreading of 1.2 to 1.5 wires per spark can be easily obtained.

Closely related to main spark spreading is the problem of photon-initiated satellite sparking. It has been found that admixtures containing small concentrations of ethanol have a high coefficient of absorbtion for spark-produced photons while having no qualities which might cause a deleterious effect on the other performance characteristics of the module. With small concentrations $(1 / 2 \%$ partial pressure), the numbers of spurious sparks are markedly reduced without seriously effecting the operating voltage or adjacent wire spreading. Higher concentrations $(1-1.5 \%)$ gain little in suppression of satellite sparking while increasing working voltage by several hundred volts and average spark spreading by about one wire. 


\section{Multiple Track Efficiency}

Certain applications of the spark chamber modules, such as the later described gamma-ray detector, need not only good efficiency for the detection of a single ionizing particle but also require good efficiency for two or more simultaneous tracks.

Since the development of the electron avalanche is a statistical process, the spark formation time for a particular gas mixture and pressure is variable depending on the initial number of electrons available at pulse time and on the magnitude of the electric field. ${ }^{2.3}$ The spark formation time for a second spark may be on the order of $10 \%$ or $15 \%$ greater than the first - if the electric field has remained undisturbed by the first spark. Since the formative time with this configuration is around $50 \mathrm{~ns}$., then for good second track efficiency the discharge time constant must be large compared to 5 or $10 \mathrm{~ns}$. To achieve this, modules intended for use in detectors requiring good double track efficiency utilize $100 \mathrm{ohm}$ resistors to terminate each cathode grid wire giving discharge time constants of 50 to 100 nanoseconds, depending on pulser circuit parameters.

\section{GAMMA-RAY DETECTOR}

A balloon-borne detector for cosmic gamma-ray point sources has been assembled from the wire grid modules just discussed (Figure 3). Results of flights with this detector are presently being analyzed and will be published at a later date.

\section{Construction}

The detector, outline shown in Figure 4, consists of two identical spark chambers, each composed of $15 \mathrm{X}-\mathrm{Y}$ modules of the type just described. The 16 th module placed at the bottom of each chamber utilizes one plane of wires strung at $45^{\circ}$ to the frame to reduce the coordinate ambiguity that exists when there are two tracks. A metal plate is used as the ground electrode on the $45^{\circ}$ modules. The decks are stacked keeping grids of equal adjacent to avoid sparking between modules.

Between each of the 32 modules is placed an electrically-free plate coated with 2.7 mils (1/50 of a radiation length) of gold, serving to convert incoming gammas into electron-positron pairs. The large number of thin interaction plates are necessary to obtain a high degree of angular resolution while maintaining a high total conversion efficiency for the detector. Information on the energy and direction of the incoming gamma may be extracted from the charged 
particle trajectories in the spark chamber. The coulomb scattering of the pair in the thin gold covered plates yields information on the energy of the incident gamma, consequently it is necessary to accurately determine the deviation of trajectory from plate to plate. This requires operating conditions that will give both high efficiency and minimal spark spreading. To insure good efficiency for registering double tracks, each of the modules utilizes $100 \mathrm{ohm}$ resistors in series with the cathode grid wires.

The sealed chamber is thoroughly evacuated and filled to 1.5 atmospheres with neon-helium, argon $(1 \%)$. ethanol $(1 / 2 \%)$ gas mixture. Pulsing the modules with a modest overvoltage $(1.7 \mathrm{kv})$ yields an average single track efficiency for the ensemble of better than $90 \%$, nearly the same for doubles, and even very good efficiency for three and four simultaneous tracks. The average spark resolution obtained under these conditions is somewhat less than two wires.

\section{$\underline{\text { High Voltage Pulser }}$}

The high voltage pulsers, enclosed in two pressure tight boxes mounted on the sides of the upper and lower chambers, consist of 16 E. G. \& G. type KN-2 Krytrons driven by an avalanche transistor circuit as shown in Figure 5.

The $1.7 \mathrm{kv}, 15$ nanosecond rise-time high voltage pulse from each tube drives only two modules, from separate discharge capacitors, to provide redundancy and a minimum of energy robbing between modules. Selected 2 N2087 transistors are used in the avalanche mode to provide a fast 800 volt trigger pulse to the Krytron grids. To avoid high voltage breakdown problems in the neon, the $2.2 \mathrm{kv}$ plate voltage supply for the discharge tubes and the 300 volt converter, which supplies both the avalanche transistor and the $150 \mu \mathrm{a}$ "keepalive" current for each $\mathrm{KN}-2$, are also contained within the nitrogen-filled pulser boxes. The small value "tuning" resistor between the output glass-metal feed-through terminal and the module grid is used for minor adjustments in the peak pulse voltage. All high current module grounds are decoupled from low level circuit common by one kilohm and returned separately to the pulser boxes with shield braid or large diameter wire to maintain low inductance.

No clearing field has been incorporated in the high voltage system; recombination and impurity attachment being adequate to clear away the ions formed by unwanted cosmic ray charged particle tracks ( 100 per second at altitude) and prevent refiring after the one second read-out busy dead time. 


\section{Coincidence Detectors}

The spark chamber is energized whenever a coincidence telescope consisting of a thin scintillator and a Cerenkov counter detect the passage of a charged particle while a surrounding plastic guard scintillator detects nothing, denoting a neutral particle which interacts within the chamber.

The central Pilot B scintillator, a five inch diameter disc 3/16 inch thick, is viewed by an RCA C-7151H photomultiplier from one edge through a light pipe made from six strips of plexiglas which are heated and individually formed into a right angle elbow then bonded to the scintillator.

The Cerenkov radiator is a one inch thick disc of plexiglas blackened on top to discriminate against upcoming cosmic ray albedo and viewed from below by a five inch diameter RCA 2065 phototube.

The bell-shaped Pilot B anticoincidence dome, which is loosely covered with aluminum foil before a light seal of black epoxy is applied to the surface, is viewed by six-gain-matched C-7151 photomultipliers mounted on the bottom ring. The output charges are summed to provide the anticoincidence signal. The loss of one of these photomultipliers, whose power supplies are independent, does not jeopardize the anticoincidence function.

\section{Coincidence Circuit}

A block diagram of the coincidence logic is shown in Figure 6 . The output charge signals from each element in the counter telescope are amplified, an amplitude discrimination performed and a gate signal generated. The resolving time of $200 \mathrm{~ns}$ was chosen to maintain a low chance triggering rate while still avoiding problems of transit time jitter in the large phototube. Coincidence defeat conditions have been incorporated as a convenience during calibration and checkout. The output of the coincidence system is inhibited while readout is busy, to avoid writing a new set of tracks before the old ones are totally read out.

\section{$\underline{\text { Data System }}^{4}$}

The gamma ray detector memory of 8192 cores is arranged in 256 words of 32 bit length. The 256 cores on each $\mathrm{X}-\mathrm{Y}$ module are interrogated sequentially by elements of a matrix of 16 current sources and 16 current sinks; the output detected by one of 32 sense amplifiers and recorded in a 32 bit shift register. The bit position in the shift register word in conjunction with the particular element in the $16 \times 16$ word select matrix uniquely specifies one 
coordinate of the spark which is recorded on a 16 track magnetic tape along with various coincidence telescope rates and housekeeping parameters.

\section{$\underline{\text { Conclusions }}$}

Experience with the spark chamber assembly in the gamma-ray detector has shown the wire-grid modules to be quite uniform in their operating characteristics. Only a small amount of tuning on a newly assembled detector array is necessary to obtain peak overall performance. Individual plane idiosyncrasies, such as preferential sparking, do not occur under normal operating conditions.

A second generation gamma-ray detector is now being developed at GSFC. It will utilize the same modular spark chamber concept but with an increase in active area of ten times the present detector. In addition to this program, the use of a wire-grid digitized spark chamber for measurement of cosmic ray electron energy spectrum is being studied.

\section{REFERENCES}

1. J. G. Rutherglen, Progress in Nuclear Physics, 10, 9 (1964)

2. J. Fischer and G. T. Zorn, Rev. Sci, Instr. 32, 499 (1961)

3. F. Schneider, "Multiple Track Efficiency of the Spark Chamber," CERN report $\mathrm{AR} /$ Int. GS/63-9

4. C. Ehrmann, IEEE Trans. Nuc. Sci., NS-13, 503 (1966) also GSFC preprint $\mathrm{X}-611-65-489$ 


\section{FIGURE CAPTIONS}

Figure 1 : $\mathrm{X}-\mathrm{Y}$ wire-grid module with core shelves attached.

Figure 2a: Module sparking efficiency in $98.5 \% \mathrm{Ne}-\mathrm{He}(9: 1), 1 \% \mathrm{~A}, 0.5 \%$ $\mathrm{C}_{2} \mathrm{H}_{5} \mathrm{OH}$ gas mixture

Figure 2b: Spark spreading with $100 \mathrm{ohm}$ grid wire termination

Figure 3 : Gamma-ray detector without cover or anticoincidence dome

Figure $4:$ Schematic view of detector system

Figure 5: Gamma-ray detector HV pulser (one of two shown)

Figure 6 : Coincidence system block diagram 
<smiles>C1#CC2=C1C2</smiles> 

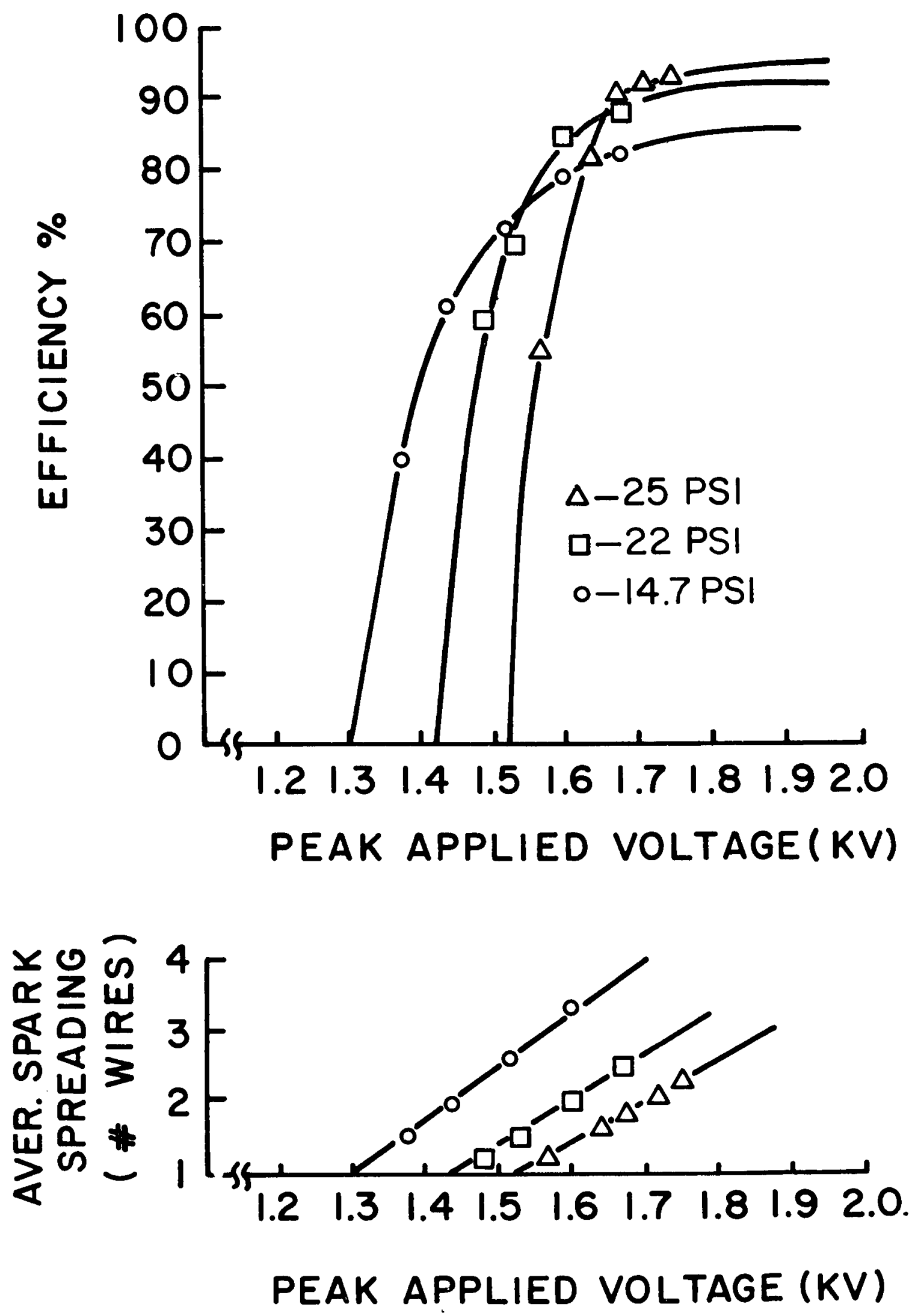

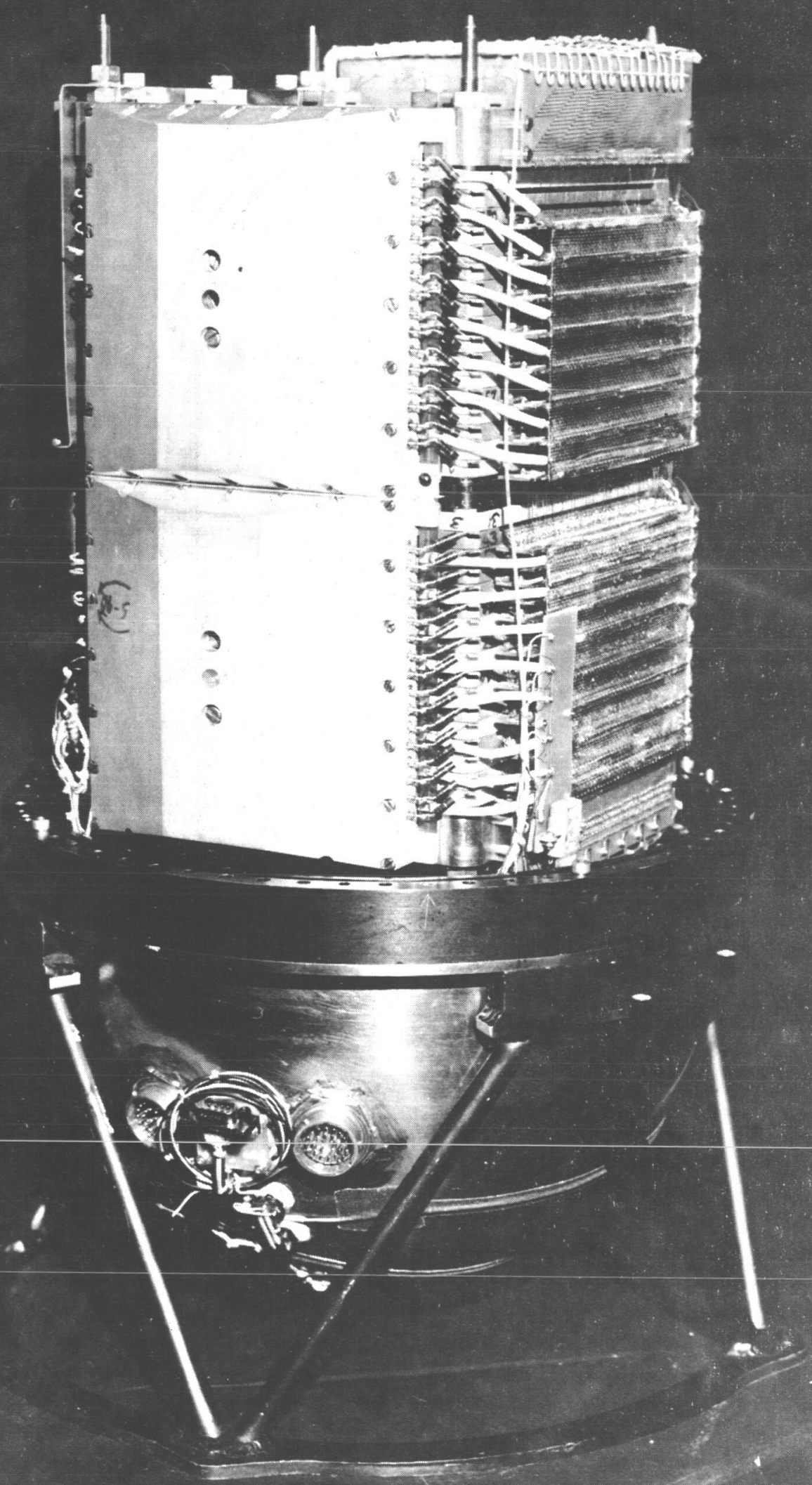


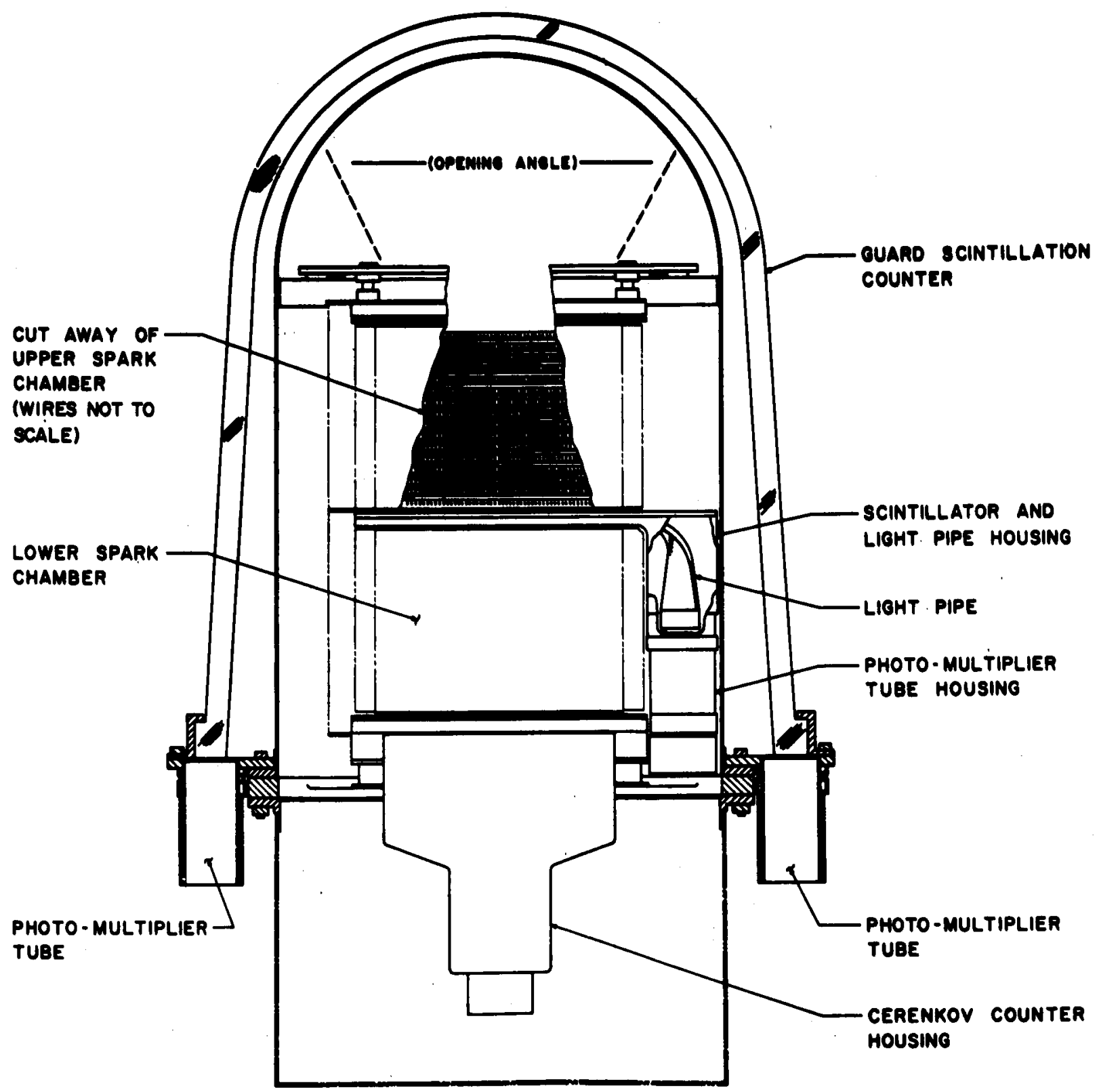


!)

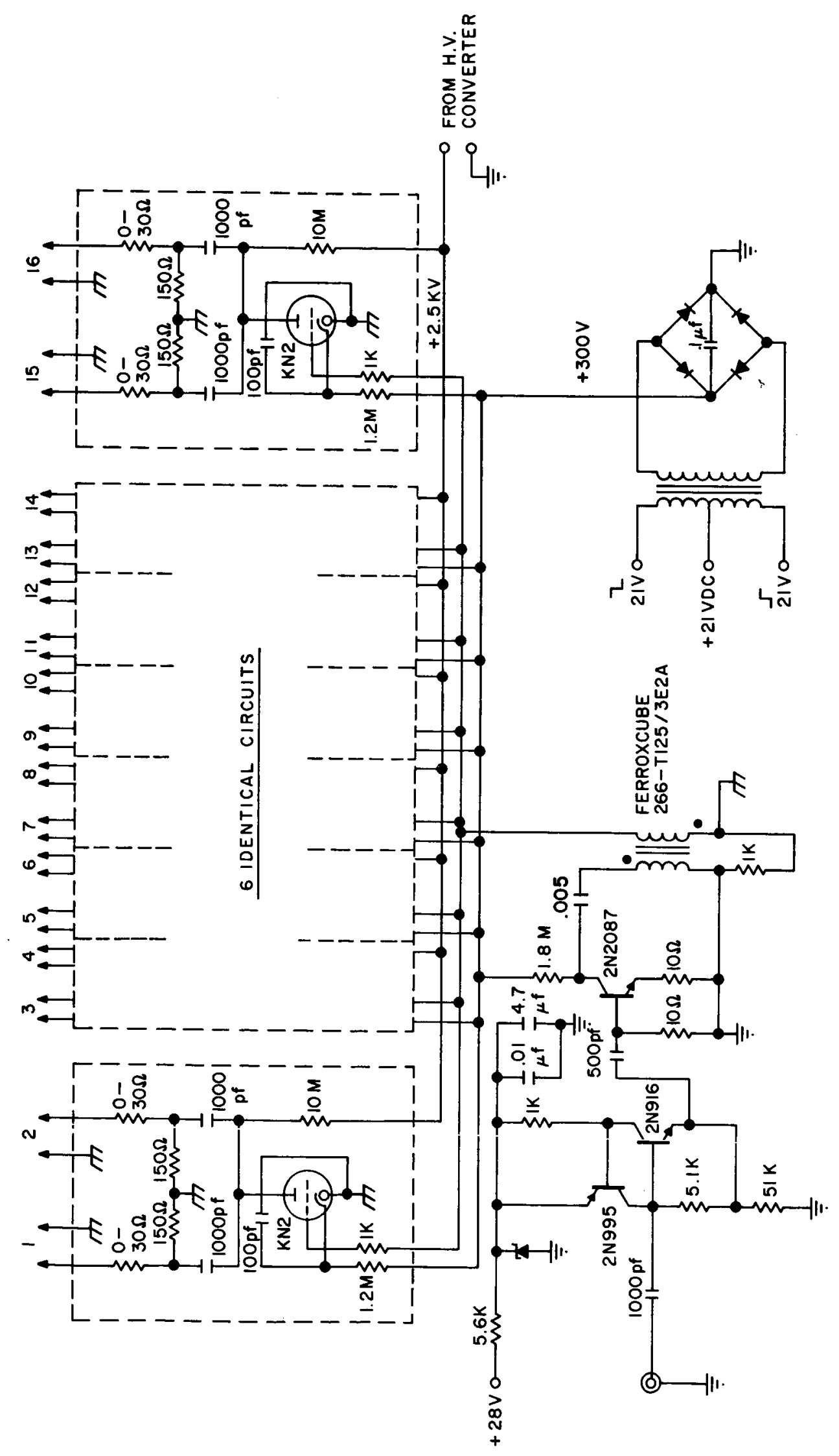




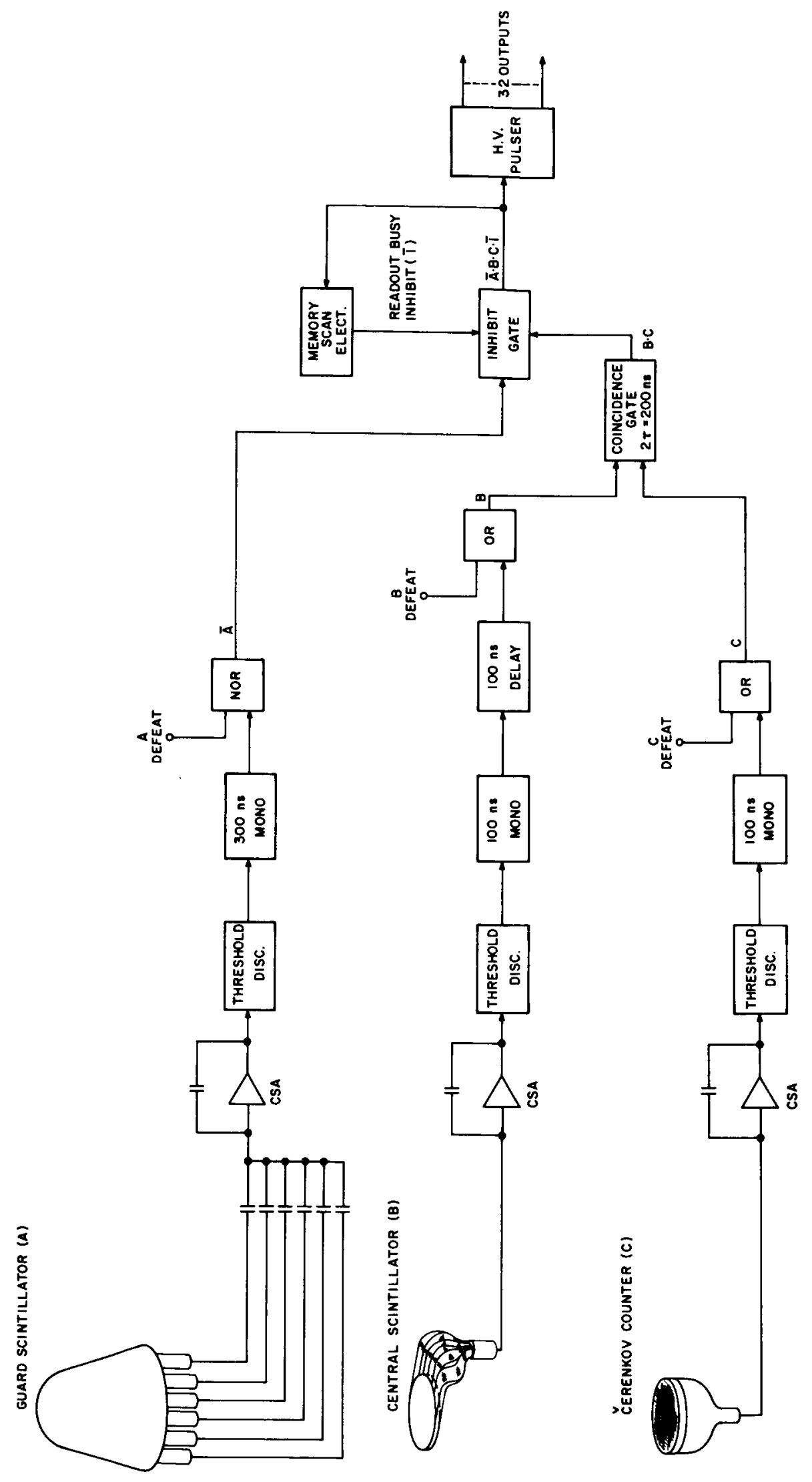

